Supporting Information

\title{
Aqueous biphasic systems as a suitable route to removal and concentrate parabens from aqueous media
}

Rodrigo Thibes Gonsalves, Fabiane Oliveira Farias, Marcos R. Mafra*, Luciana IgarashiMafra

Department of Chemical Engineering, Federal University of Paraná (UFPR), Polytechnic Center, 81531-990, Curitiba-PR, Brazil.

*Corresponding Author: Tel.: +55-41-33613586;

E-mail address: marcos.mafra@ufpr.br 
Table S1. Experimental binodal weight fraction (wt \%) data for the systems composed PEG $6000+\left(\mathrm{NH}_{4}\right)_{2} \mathrm{SO}_{4}+$ water binodal data at $298.15 \mathrm{~K}$ and atmospheric pressure ${ }^{\text {a }}$.

\begin{tabular}{cccccc}
\hline $\begin{array}{c}\text { Salt } \\
(\mathbf{w t} \%)\end{array}$ & $\begin{array}{c}\text { PEG 6000 } \\
(\mathbf{w t} \%)\end{array}$ & $\begin{array}{c}\text { Salt } \\
\mathbf{( w t} \%)\end{array}$ & $\begin{array}{c}\text { PEG 6000 } \\
\mathbf{( w t} \%)\end{array}$ & $\begin{array}{c}\text { Salt } \\
(\mathbf{w t} \%)\end{array}$ & $\begin{array}{c}\text { PEG 6000 } \\
(\mathbf{w t} \%)\end{array}$ \\
\hline 1.8803 & 50.9104 & 6.7621 & 18.7563 & 10.6245 & 6.4912 \\
2.1702 & 47.2304 & 6.9138 & 17.9487 & 10.8621 & 5.8254 \\
2.6160 & 44.0800 & 7.3268 & 16.6511 & 11.2206 & 5.0605 \\
2.8201 & 41.9101 & 7.5003 & 15.8337 & 11.7674 & 3.4958 \\
3.0811 & 38.6908 & 7.7568 & 15.0143 & 12.6628 & 2.1303 \\
3.6317 & 35.7884 & 8.0863 & 13.9725 & 13.6722 & 0.9913 \\
3.9517 & 32.9879 & 8.6169 & 12.4265 & 14.9087 & 0.4856 \\
4.4427 & 30.2413 & 8.9193 & 11.4686 & 15.7543 & 0.2983 \\
4.7289 & 28.2321 & 9.3617 & 10.1486 & 16.5984 & 0.2170 \\
5.2380 & 25.6927 & 9.5736 & 9.4320 & 18.3649 & 0.1983 \\
5.4790 & 24.1348 & 9.8431 & 8.7684 & 19.5971 & 0.1897 \\
5.7391 & 23.0489 & 10.0194 & 8.1432 & 20.3654 & 0.1558 \\
6.0208 & 21.5188 & 10.2759 & 7.5276 & 21.6490 & 0.0022 \\
6.4152 & 20.0742 & 10.4077 & 7.0804 & & \\
\hline
\end{tabular}

${ }^{\text {a }}$ Standard uncertainty $\mathrm{u}$ are $\mathrm{u}(\mathrm{w})=0.1 \%, \mathrm{u}(\mathrm{T})=0.1 \mathrm{~K}$, and $\mathrm{u}(\mathrm{P})=10 \mathrm{kPa}$. 
Table S2. Experimental binodal weight fraction (wt \%) data for the systems composed PEG $2000+\left(\mathrm{NH}_{4}\right)_{2} \mathrm{SO}_{4}+$ water binodal data at $298.15 \mathrm{~K}$ and atmospheric pressure $^{\mathrm{a}}$.

\begin{tabular}{cccccc}
\hline $\begin{array}{c}\text { Salt } \\
\mathbf{( w t} \%)\end{array}$ & $\begin{array}{c}\text { PEG 2000 } \\
\mathbf{( w t} \%)\end{array}$ & $\begin{array}{c}\text { Salt } \\
\mathbf{( w t} \%)\end{array}$ & $\begin{array}{c}\text { PEG 2000 } \\
\mathbf{( w t} \%)\end{array}$ & $\begin{array}{c}\text { Salt } \\
\mathbf{( w t} \%)\end{array}$ & $\begin{array}{c}\text { PEG 2000 } \\
\text { (wt \%) }\end{array}$ \\
\hline 1.8537 & 52.1060 & 9.7990 & 15.6268 & 14.2782 & 5.3113 \\
2.5425 & 45.8411 & 10.7804 & 13.0531 & 15.1645 & 3.9845 \\
3.7711 & 38.8437 & 10.9705 & 12.3773 & 15.9034 & 3.0370 \\
4.6869 & 34.0680 & 11.5860 & 11.2921 & 17.6171 & 1.6998 \\
5.2817 & 30.8892 & 11.9970 & 9.9869 & 18.2985 & 1.2930 \\
6.4188 & 26.1837 & 12.1833 & 9.7324 & 19.5047 & 0.7824 \\
7.1992 & 23.5141 & 12.4813 & 9.0589 & 20.5575 & 0.4369 \\
7.9661 & 20.7499 & 13.0539 & 7.6832 & 22.3567 & 0.1065 \\
8.8469 & 17.4842 & 13.4285 & 6.9278 & 24.5124 & 0.1605 \\
9.0909 & 16.8628 & 13.4838 & 6.9832 & 26.6415 & 0.1065 \\
9.3882 & 16.7353 & 13.8444 & 6.0860 & 29.8001 & 0.1065 \\
\hline
\end{tabular}

${ }^{a}$ Standard uncertainty $\mathrm{u}$ are $\mathrm{u}(\mathrm{w})=0.1 \%, \mathrm{u}(\mathrm{T})=0.1 \mathrm{~K}$, and $\mathrm{u}(\mathrm{P})=10 \mathrm{kPa}$. 
Table S3. Experimental binodal weight fraction (wt \%) data for the systems composed PEG $400+\left(\mathrm{NH}_{4}\right)_{2} \mathrm{SO}_{4}+$ water binodal data at $298.15 \mathrm{~K}$ and atmospheric pressure $^{\text {a }}$

\begin{tabular}{cccccc}
\hline $\begin{array}{c}\text { Salt } \\
\mathbf{( w t} \%)\end{array}$ & $\begin{array}{c}\text { PEG 400 } \\
\mathbf{( w t} \%)\end{array}$ & $\begin{array}{c}\text { Salt } \\
\mathbf{( w t} \%)\end{array}$ & $\begin{array}{c}\text { PEG 400 } \\
\mathbf{( w t} \%)\end{array}$ & $\begin{array}{c}\text { Salt } \\
\mathbf{( w t} \%)\end{array}$ & $\begin{array}{c}\text { PEG 400 } \\
\mathbf{( w t} \%)\end{array}$ \\
\hline 2.8959 & 55.6808 & 6.1048 & 39.7832 & 14.1600 & 22.5996 \\
3.2395 & 53.1920 & 6.7202 & 37.8702 & 15.3498 & 20.1343 \\
3.4647 & 51.2703 & 7.2869 & 35.9977 & 16.1056 & 18.9805 \\
3.7169 & 49.3987 & 7.4610 & 35.8316 & 16.9140 & 17.6611 \\
3.7981 & 49.4367 & 8.4531 & 33.3409 & 17.6583 & 15.8084 \\
4.1109 & 47.5995 & 9.2907 & 31.0286 & 19.2386 & 14.9635 \\
4.2964 & 46.8616 & 10.0460 & 29.6134 & 20.1768 & 12.0958 \\
4.7087 & 44.8861 & 10.9165 & 27.8009 & 22.0220 & 8.7083 \\
5.1905 & 43.2477 & 11.3322 & 27.3243 & 23.7100 & 6.8556 \\
5.2092 & 42.8565 & 11.9895 & 25.7428 & 26.5125 & 5.0887 \\
5.5686 & 41.6622 & 12.9474 & 24.2713 & & \\
6.0810 & 39.9773 & 13.4740 & 23.4022 & & \\
\hline
\end{tabular}

${ }^{a}$ Standard uncertainty $\mathrm{u}$ are $\mathrm{u}(\mathrm{w})=0.1 \%, \mathrm{u}(\mathrm{T})=0.1 \mathrm{~K}$, and $\mathrm{u}(\mathrm{P})=10 \mathrm{kPa}$. 
Table S4. Experimental binodal weight fraction (wt \%) data for the systems composed PPG $400+\left(\mathrm{NH}_{4}\right)_{2} \mathrm{SO}_{4}+$ water binodal data at $298.15 \mathrm{~K}$ and atmospheric pressure $^{\mathrm{a}}$.

\begin{tabular}{|c|c|c|c|c|c|}
\hline $\begin{array}{c}\text { Salt } \\
(w t \%)\end{array}$ & $\begin{array}{c}\text { PPG } 400 \\
\text { (wt \%) }\end{array}$ & $\begin{array}{c}\text { Salt } \\
(\text { wt \%) }\end{array}$ & $\begin{array}{c}\text { PPG 400 } \\
(w t \%)\end{array}$ & $\begin{array}{c}\text { Salt } \\
(\text { wt \%) }\end{array}$ & $\begin{array}{r}\text { PPG 400 } \\
(w t \%)\end{array}$ \\
\hline 0.5065 & 92.1980 & 2.3644 & 32.7910 & 6.4132 & 10.7272 \\
\hline 0.5066 & 87.2079 & 2.5826 & 31.5026 & 6.4621 & 10.2476 \\
\hline 0.5954 & 79.1489 & 2.9655 & 28.0483 & 6.6201 & 10.0780 \\
\hline 0.6580 & 74.6390 & 3.2327 & 26.0921 & 6.7631 & 9.5534 \\
\hline 0.7782 & 69.5155 & 3.3183 & 24.5415 & 6.8934 & 9.0491 \\
\hline 0.7782 & 66.4199 & 3.6579 & 22.9337 & 7.0331 & 8.9803 \\
\hline 0.8408 & 63.0042 & 3.8420 & 21.2115 & 7.2186 & 8.4627 \\
\hline 0.9609 & 60.6826 & 3.9996 & 19.9646 & 7.4387 & 7.9191 \\
\hline 1.1202 & 59.9550 & 4.3701 & 18.3727 & 7.9270 & 7.0505 \\
\hline 1.1397 & 57.0527 & 4.5507 & 17.3011 & 8.4830 & 6.3700 \\
\hline 1.2541 & 52.8642 & 4.7667 & 15.8007 & 9.1628 & 4.5170 \\
\hline 1.4200 & 49.4911 & 5.0025 & 14.8829 & 9.9569 & 3.4973 \\
\hline 1.5052 & 47.6787 & 5.1659 & 13.9901 & 11.0392 & 2.4096 \\
\hline 1.5686 & 47.3277 & 5.2399 & 13.9175 & 12.8543 & 1.1962 \\
\hline 1.7185 & 44.9014 & 5.4508 & 13.0635 & 15.2966 & 0.3869 \\
\hline 1.8993 & 42.0281 & 5.6219 & 12.7137 & 19.1994 & 0.0361 \\
\hline 1.9534 & 40.4308 & 5.7216 & 12.1751 & 22.6272 & 0.0022 \\
\hline 2.0993 & 37.6996 & 6.1966 & 11.2760 & & \\
\hline 2.1871 & 36.4359 & 6.0487 & 11.2421 & & \\
\hline
\end{tabular}

${ }^{a}$ Standard uncertainty $\mathrm{u}$ are $\mathrm{u}(\mathrm{w})=0.1 \%, \mathrm{u}(\mathrm{T})=0.1 \mathrm{~K}$, and $\mathrm{u}(\mathrm{P})=10 \mathrm{kPa}$. 
Table S5. Critical point of systems composed of $\left(\mathrm{NH}_{4}\right)_{2} \mathrm{SO}_{4}+$ polymer + water at 298.15

$\mathrm{K}$ and atmospheric pressure.

\begin{tabular}{|c|c|c|c|c|c|}
\hline \multirow[t]{2}{*}{ System } & \multirow[t]{2}{*}{$f$} & \multirow[t]{2}{*}{$\boldsymbol{g}$} & \multirow[t]{2}{*}{$\mathbf{R}^{2}$} & \multicolumn{2}{|c|}{$\begin{array}{c}\text { Critical point } \\
\text { (wt \%) }\end{array}$} \\
\hline & & & & $\mathbf{X}$ & $\mathbf{Y}$ \\
\hline$\left(\mathrm{NH}_{4}\right)_{2} \mathrm{SO}_{4}+$ PEG $6000+$ water & 2.6742 & -9.6457 & 0.9777 & 8.3713 & 12.7406 \\
\hline$\left(\mathrm{NH}_{4}\right)_{2} \mathrm{SO}_{4}+$ PEG $2000+$ water & 2.5284 & -12.282 & 0.9805 & 10.3493 & 13.8851 \\
\hline$\left(\mathrm{NH}_{4}\right)_{2} \mathrm{SO}_{4}+\mathrm{PEG} 400+$ water & 1.2589 & 4.4228 & 0.9926 & 13.9429 & 21.9753 \\
\hline$\left(\mathrm{NH}_{4}\right)_{2} \mathrm{SO}_{4}+\mathrm{PPG} 400+$ water & 1.7046 & 60.6830 & 0.9936 & 0.9900 & 62.3702 \\
\hline
\end{tabular}


Figure S1. Tie-lines and overall compostions of each system composed of polymer + $\left(\mathrm{NH}_{4}\right)_{2} \mathrm{SO}_{4}+$ water appliyng for the partition assays.
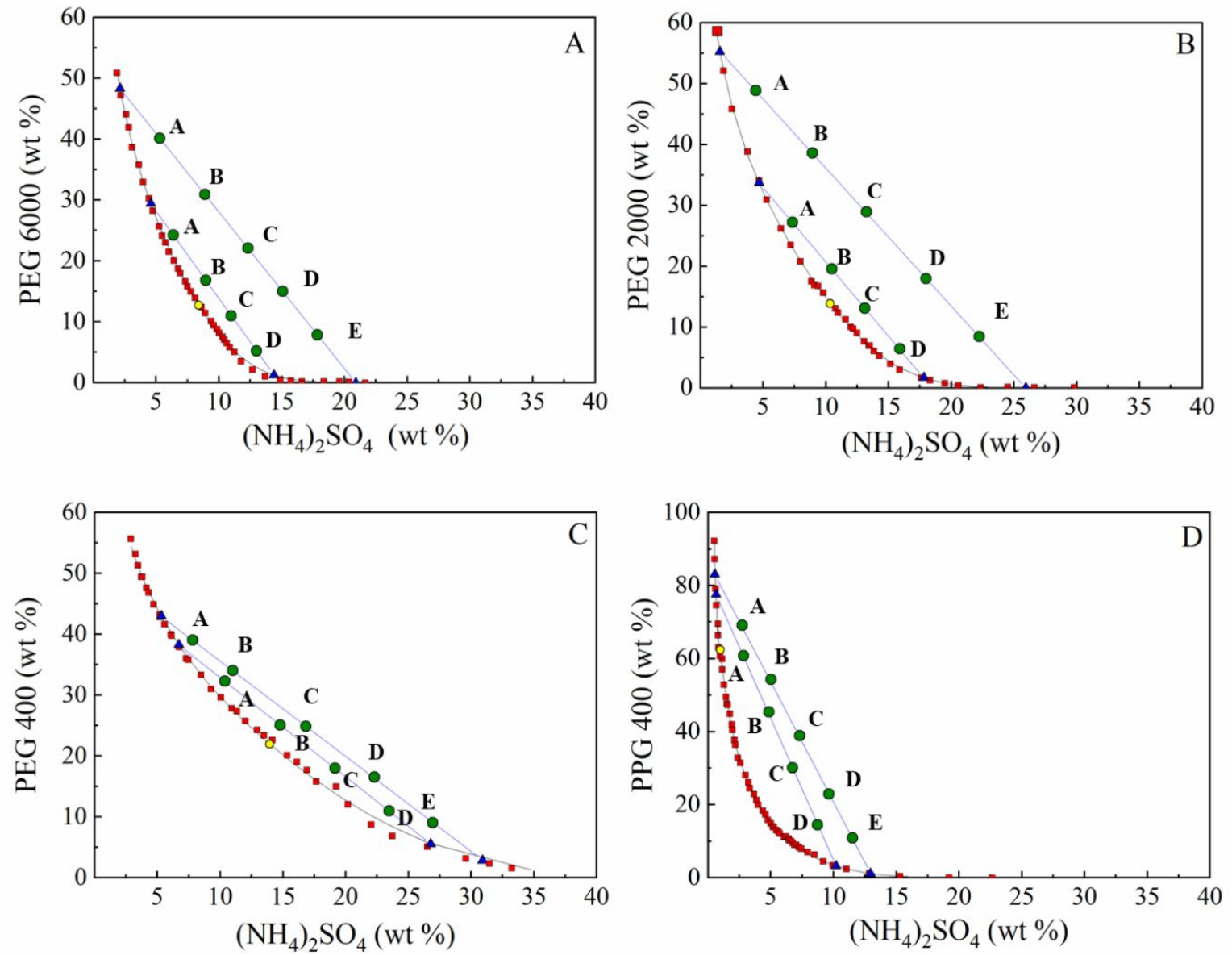
Figure S2. Experimental and estimated tie-lines for the systems: (A) PEG $6000+\left(\mathrm{NH}_{4}\right)_{2} \mathrm{SO}_{4}+\mathrm{H}_{2} \mathrm{O}$, (B) PEG $2000+\left(\mathrm{NH}_{4}\right)_{2} \mathrm{SO}_{4}+\mathrm{H}_{2} \mathrm{O},(\mathrm{C}) \mathrm{PEG} 400+\left(\mathrm{NH}_{4}\right)_{2} \mathrm{SO}_{4}+\mathrm{H}_{2} \mathrm{O}$ and (D) PPG $400+\left(\mathrm{NH}_{4}\right)_{2} \mathrm{SO}_{4}+\mathrm{H}_{2} \mathrm{O}$ at $298.15 \mathrm{~K}$ and atmospheric pressure.
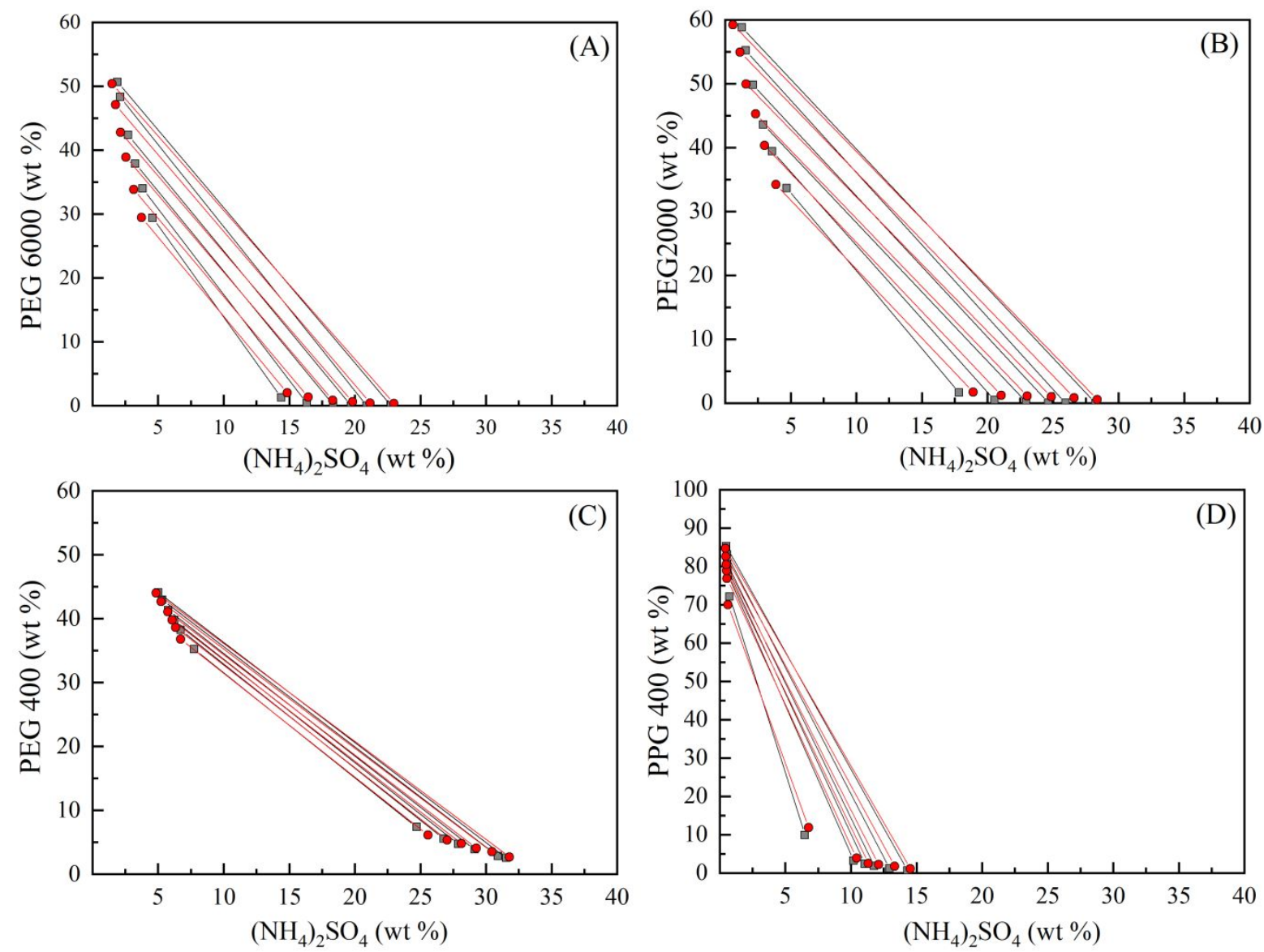

$\longrightarrow$ - Experimental tie-line $\longrightarrow$ NRTL correlated tie-line 
Table S6. $\mathrm{pH}$ measurement of the tie-lines for the $\left(\mathrm{NH}_{4}\right)_{2} \mathrm{SO}_{4}+$ polymer + water systems at $298.15 \mathrm{~K}$ and atmospheric pressure ${ }^{\mathrm{a}}$.

\begin{tabular}{lcc}
\hline \multicolumn{1}{c}{ System } & $\mathbf{p H}$ polymer-rich phase & pH salt-rich phase \\
\hline$\left(\mathrm{NH}_{4}\right)_{2} \mathrm{SO}_{4}+$ PEG 6000 + water & $5.44 \pm 0.02$ & $5.20 \pm 0.01$ \\
$\left(\mathrm{NH}_{4}\right)_{2} \mathrm{SO}_{4}+$ PEG 2000 + water & $4.90 \pm 0.05$ & $5.04 \pm 0.03$ \\
$\left(\mathrm{NH}_{4}\right)_{2} \mathrm{SO}_{4}+$ PEG 400 + water & $4.58 \pm 0.13$ & $4.48 \pm 0.07$ \\
$\left(\mathrm{NH}_{4}\right)_{2} \mathrm{SO}_{4}+$ PPG 400 + water & $4.07 \pm 0.08$ & $4.03 \pm 0.16$ \\
\hline
\end{tabular}

${ }^{\mathrm{a}}$ Standard uncertainty $\mathrm{u}(\mathrm{pH})=0.01$ and $\mathrm{u}(\mathrm{P})=10 \mathrm{kPa}$. 
Table S7. Partition coefficient (K) and extraction efficiency (EE\%) for methyl- (MeP), ethyl- (EtP), propyl- (PrP) e butylparaben (BuP) for PEG6000 + $\left(\mathrm{NH}_{4}\right)_{2} \mathrm{SO}_{4}+\mathrm{H}_{2} \mathrm{O}$ and $\mathrm{PEG} 2000+\left(\mathrm{NH}_{4}\right)_{2} \mathrm{SO}_{4}+\mathrm{H}_{2} \mathrm{O}$.

\begin{tabular}{|c|c|c|c|c|c|c|c|c|c|}
\hline \multirow{2}{*}{\multicolumn{2}{|c|}{ TL }} & \multicolumn{4}{|c|}{$\bar{K}$} & \multicolumn{4}{|c|}{$\overline{E E}(\%)$} \\
\hline & & MeP & EtP & PrP & BuP & MeP & EtP & PrP & BuP \\
\hline \multicolumn{10}{|c|}{$\mathrm{PEG6000}+\left(\mathrm{NH}_{4}\right)_{2} \mathrm{SO}_{4}+\mathrm{H}_{2} \mathrm{O}$} \\
\hline \multirow{4}{*}{ TL I } & 1 & $18.52 \pm 1.45$ & $24.46 \pm 1.25$ & $108.68 \pm 0.62$ & $69.98 \pm 0.48$ & $98.51 \pm 0.65$ & $98.99 \pm 0.24$ & $99.73 \pm 0.19$ & $99.61 \pm 0.05$ \\
\hline & 2 & $13.60 \pm 0.17$ & $25.00 \pm 0.54$ & $38.61 \pm 0.16$ & $51.68 \pm 1.25$ & $94.28 \pm 0.52$ & $97.51 \pm 0.95$ & $98.10 \pm 0.16$ & $98.41 \pm 0.19$ \\
\hline & 3 & $13.35 \pm 0.59$ & $31.95 \pm 0.15$ & $28.98 \pm 0.94$ & $42.90 \pm 1.26$ & $87.89 \pm 0.82$ & $95.70 \pm 0.52$ & $94.07 \pm 1.21$ & $96.05 \pm 0.20$ \\
\hline & 4 & $11.11 \pm 1.76$ & $33.44 \pm 1.59$ & $19.60 \pm 0.48$ & $31.55 \pm 0.21$ & $62.44 \pm 0.09$ & $89.72 \pm 0.55$ & $80.65 \pm 0.96$ & $83.36 \pm 0.18$ \\
\hline \multirow{5}{*}{ TL II } & 1 & $442.34 \pm 1.55$ & $88.42 \pm 1.32$ & $495.34 \pm 0.51$ & $150.10 \pm 0.29$ & $99.95 \pm 0.48$ & $99.77 \pm 0.28$ & $99.95 \pm 0.45$ & $99.86 \pm 0.51$ \\
\hline & 2 & $65.72 \pm 1.13$ & $84.99 \pm 0.12$ & $1173.09 \pm 1.48$ & $228.90 \pm 1.95$ & $99.26 \pm 0.19$ & $99.47 \pm 0.12$ & $99.69 \pm 0.74$ & $99.78 \pm 0.41$ \\
\hline & 3 & $26.21 \pm 0.76$ & $114.52 \pm 1.00$ & $102.08 \pm 1.69$ & $211.84 \pm 0.66$ & $95.62 \pm 0.62$ & $99.00 \pm 0.75$ & $98.91 \pm 0.05$ & $99.46 \pm 0.01$ \\
\hline & 4 & $52.93 \pm 0.96$ & $63.13 \pm 1.16$ & $98.60 \pm 0.12$ & $307.79 \pm 0.19$ & $95.80 \pm 0.19$ & $96.92 \pm 0.89$ & $97.90 \pm 0.26$ & $99.31 \pm 0.95$ \\
\hline & 5 & $50.35 \pm 0.86$ & $17.06 \pm 1.66$ & $97.59 \pm 0.49$ & $183.77 \pm 0.13$ & $90.19 \pm 0.48$ & $81.55 \pm 0.17$ & $95.32 \pm 0.95$ & $97.65 \pm 1.11$ \\
\hline \multicolumn{10}{|c|}{$\mathrm{PEG2000}+\left(\mathrm{NH}_{4}\right)_{2} \mathrm{SO}_{4}+\mathrm{H}_{2} \mathrm{O}$} \\
\hline \multirow{4}{*}{ TL I } & 1 & $56.69 \pm 0.02$ & $36.26 \pm 0.48$ & $79.77 \pm 0.08$ & $116.37 \pm 1.52$ & $99.40 \pm 0.95$ & $99.26 \pm 0.15$ & $99.63 \pm 1.00$ & $99.79 \pm 0.84$ \\
\hline & 2 & $26.26 \pm 0.15$ & $33.64 \pm 0.95$ & $76.27 \pm 0.05$ & $110.31 \pm 0.79$ & $96.73 \pm 0.18$ & $98.73 \pm 0.59$ & $98.87 \pm 1.15$ & $99.36 \pm 0.25$ \\
\hline & 3 & $20.84 \pm 0.22$ & $27.08 \pm 1.00$ & $73.70 \pm 0.15$ & $66.30 \pm 0.48$ & $92.08 \pm 0.25$ & $94.27 \pm 1.32$ & $97.27 \pm 0.65$ & $97.63 \pm 0.15$ \\
\hline & 4 & $21.81 \pm 0.52$ & $7.34 \pm 0.96$ & $32.25 \pm 0.58$ & $37.74 \pm 0.75$ & $80.93 \pm 0.54$ & $63.74 \pm 0.12$ & $87.74 \pm 0.48$ & $85.60 \pm 1.49$ \\
\hline \multirow{5}{*}{ TL II } & 1 & $37.84 \pm 0.23$ & $62.16 \pm 1.89$ & $45.68 \pm 0.85$ & $790.77 \pm 0.55$ & $99.62 \pm 0.84$ & $99.79 \pm 1.00$ & $99.72 \pm 0.65$ & $99.98 \pm 0.95$ \\
\hline & 2 & $56.16 \pm 0.75$ & $101.37 \pm 0.84$ & $60.82 \pm 0.68$ & $79.19 \pm 0.02$ & $99.33 \pm 0.25$ & $99.65 \pm 0.95$ & $99.39 \pm 0.47$ & $99.50 \pm 0.18$ \\
\hline & 3 & $118.03 \pm 0.59$ & $140.03 \pm 0.25$ & $84.34 \pm 0.94$ & $132.82 \pm 0.29$ & $99.33 \pm 0.15$ & $99.44 \pm 0.89$ & $99.07 \pm 0.15$ & $99.39 \pm 0.25$ \\
\hline & 4 & $1213.96 \pm 1.85$ & $163.63 \pm 0.15$ & $356.33 \pm 0.95$ & $228.59 \pm 0.21$ & $99.82 \pm 0.49$ & $98.89 \pm 1.00$ & $99.43 \pm 1.09$ & $99.15 \pm 0.54$ \\
\hline & 5 & $127.94 \pm 1.01$ & $84.65 \pm 1.49$ & $1173.16 \pm 1.52$ & $251.38 \pm 0.61$ & $96.32 \pm 0.95$ & $93.65 \pm 0.15$ & $99.55 \pm 1.02$ & $97.99 \pm 0.68$ \\
\hline
\end{tabular}


Table S8. Partition coefficient $(K)$ and extraction efficiency $(E E \%)$ for methyl- (MeP), ethyl- (EtP), propyl- $(\operatorname{PrP})$ e butylparaben $(\mathrm{BuP})$ for PEG400 + $\left(\mathrm{NH}_{4}\right)_{2} \mathrm{SO}_{4}+\mathrm{H}_{2} \mathrm{O}$ and $\mathrm{PPG} 400+\left(\mathrm{NH}_{4}\right)_{2} \mathrm{SO}_{4}+\mathrm{H}_{2} \mathrm{O}$.

\begin{tabular}{|c|c|c|c|c|c|c|c|c|c|}
\hline \multirow{2}{*}{\multicolumn{2}{|c|}{ TL }} & \multicolumn{4}{|c|}{$\boldsymbol{K}$} & \multicolumn{4}{|c|}{$\boldsymbol{E} \boldsymbol{E}(\%)$} \\
\hline & & MeP & EtP & PrP & BuP & MeP & EtP & PrP & BuP \\
\hline \multicolumn{10}{|c|}{$\mathrm{PEG400}+\left(\mathrm{NH}_{4}\right)_{2} \mathrm{SO}_{4}+\mathrm{H}_{2} \mathrm{O}$} \\
\hline \multirow{4}{*}{ TL I } & 1 & $16.48 \pm 0.05$ & $24.46 \pm 0.49$ & $23.63 \pm 0.05$ & $29.14 \pm 1.95$ & $99.53 \pm 0.48$ & $98.99 \pm 0.24$ & $99.15 \pm 0.25$ & $99.21 \pm 0.20$ \\
\hline & 2 & $17.59 \pm 0.19$ & $25.00 \pm 0.65$ & $65.92 \pm 0.89$ & $40.03 \pm 1.29$ & $96.81 \pm 0.28$ & $97.51 \pm 0.19$ & $99.06 \pm 0.12$ & $98.50 \pm 0.75$ \\
\hline & 3 & $20.01 \pm 0.19$ & $31.95 \pm 0.19$ & $30.87 \pm 0.18$ & $45.42 \pm 1.51$ & $93.44 \pm 0.94$ & $95.70 \pm 0.65$ & $95.54 \pm 0.05$ & $96.95 \pm 0.03$ \\
\hline & 4 & $23.45 \pm 0.18$ & $33.44 \pm 0.41$ & $64.20 \pm 1.25$ & $57.11 \pm 0.95$ & $84.94 \pm 0.65$ & $89.72 \pm 0.18$ & $94.31 \pm 0.78$ & $93.33 \pm 0.29$ \\
\hline \multirow{5}{*}{ TL II } & 1 & $27.98 \pm 0.53$ & $35.39 \pm 0.19$ & $84.59 \pm 0.19$ & $62.72 \pm 0.63$ & $99.63 \pm 0.21$ & $99.69 \pm 1.09$ & $99.87 \pm 1.15$ & $99.81 \pm 0.94$ \\
\hline & 2 & $28.01 \pm 1.89$ & $44.79 \pm 0.51$ & $1127.87 \pm 1.19$ & $38.00 \pm 1.15$ & $98.99 \pm 0.61$ & $99.31 \pm 0.29$ & $99.97 \pm 0.25$ & $99.24 \pm 1.19$ \\
\hline & 3 & $66.75 \pm 0.84$ & $77.85 \pm 0.22$ & $92.80 \pm 0.17$ & $66.84 \pm 1.25$ & $98.75 \pm 0.87$ & $98.95 \pm 1.05$ & $99.13 \pm 0.31$ & $98.66 \pm 0.11$ \\
\hline & 4 & $57.86 \pm 0.19$ & $88.14 \pm 0.02$ & $231.28 \pm 0.15$ & $89.04 \pm 1.22$ & $96.71 \pm 0.78$ & $96.60 \pm 0.92$ & $99.19 \pm 0.54$ & $98.44 \pm 0.13$ \\
\hline & 5 & $82.45 \pm 0.52$ & $96.42 \pm 0.62$ & $154.46 \pm 0.11$ & $176.81 \pm 0.55$ & $94.18 \pm 0.95$ & $95.03 \pm 0.62$ & $96.36 \pm 0.98$ & $96.78 \pm 1.42$ \\
\hline \multicolumn{10}{|c|}{$\mathrm{PPG400}+\left(\mathrm{NH}_{4}\right)_{2} \mathrm{SO}_{4}+\mathrm{H}_{2} \mathrm{O}$} \\
\hline \multirow{4}{*}{ TL I } & 1 & $19.13 \pm 0.05$ & $16.64 \pm 0.82$ & $18.31 \pm 0.32$ & $16.88 \pm 1.98$ & $98.70 \pm 0.19$ & $98.54 \pm 0.17$ & $98.68 \pm 0.45$ & $98.61 \pm 0.74$ \\
\hline & 2 & $45.21 \pm 0.26$ & $26.33 \pm 0.09$ & $52.84 \pm 0.46$ & $22.49 \pm 0.61$ & $98.70 \pm 0.25$ & $97.73 \pm 0.15$ & $98.84 \pm 0.21$ & $97.44 \pm 0.95$ \\
\hline & 3 & $55.75 \pm 0.95$ & $60.77 \pm 0.95$ & $135.85 \pm 1.21$ & $78.98 \pm 1.76$ & $96.92 \pm 0.18$ & $97.40 \pm 0.11$ & $98.72 \pm 0.12$ & $97.78 \pm 0.38$ \\
\hline & 4 & $55.88 \pm 0.52$ & $82.19 \pm 0.19$ & $118.68 \pm 0.12$ & $145.00 \pm 0.94$ & $81.57 \pm 0.89$ & $87.03 \pm 0.32$ & $91.08 \pm 0.29$ & $93.21 \pm 0.41$ \\
\hline \multirow{5}{*}{ TL II } & 1 & $81.13 \pm 0.89$ & $16.15 \pm 0.09$ & $29.27 \pm 1.29$ & $24.54 \pm 1.19$ & $99.86 \pm 0.44$ & $99.23 \pm 0.22$ & $99.72 \pm 0.06$ & $99.76 \pm 0.01$ \\
\hline & 2 & $59.14 \pm 1.20$ & $24.33 \pm 1.12$ & $47.90 \pm 1.42$ & $30.87 \pm 0.11$ & $99.30 \pm 0.17$ & $98.39 \pm 0.09$ & $99.15 \pm 0.25$ & $98.69 \pm 0.15$ \\
\hline & 3 & $199.22 \pm 1.13$ & $56.37 \pm 1.11$ & $129.24 \pm 0.39$ & $63.61 \pm 1.13$ & $99.49 \pm 0.58$ & $98.27 \pm 0.25$ & $99.23 \pm 0.12$ & $98.44 \pm 0.21$ \\
\hline & 4 & $265.92 \pm 1.11$ & $84.54 \pm 0.95$ & $217.60 \pm 0.95$ & $112.31 \pm 0.06$ & $98.78 \pm 0.75$ & $96.84 \pm 0.65$ & $98.59 \pm 0.11$ & $97.36 \pm 0.52$ \\
\hline & 5 & $185.00 \pm 0.20$ & $218.60 \pm 0.18$ & $555.35 \pm 0.75$ & $533.75 \pm 0.33$ & $94.21 \pm 0.19$ & $94.93 \pm 1.12$ & $98.52 \pm 1.25$ & $98.36 \pm 0.22$ \\
\hline
\end{tabular}


Figure S3. (a) Logarithmic function of partition coefficients and (b) extraction efficiency of a mixture of parabens in ABS composed of different polymers, salt and water at $298.2 \mathrm{~K}$ and atmospheric pressure

(a)

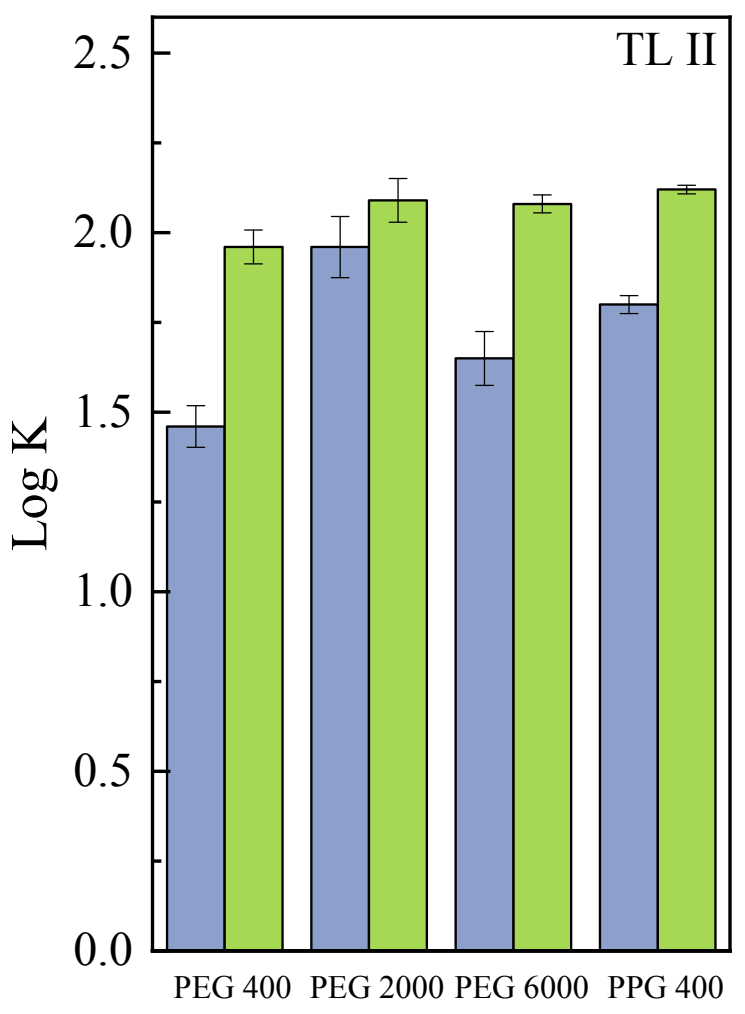

(b)

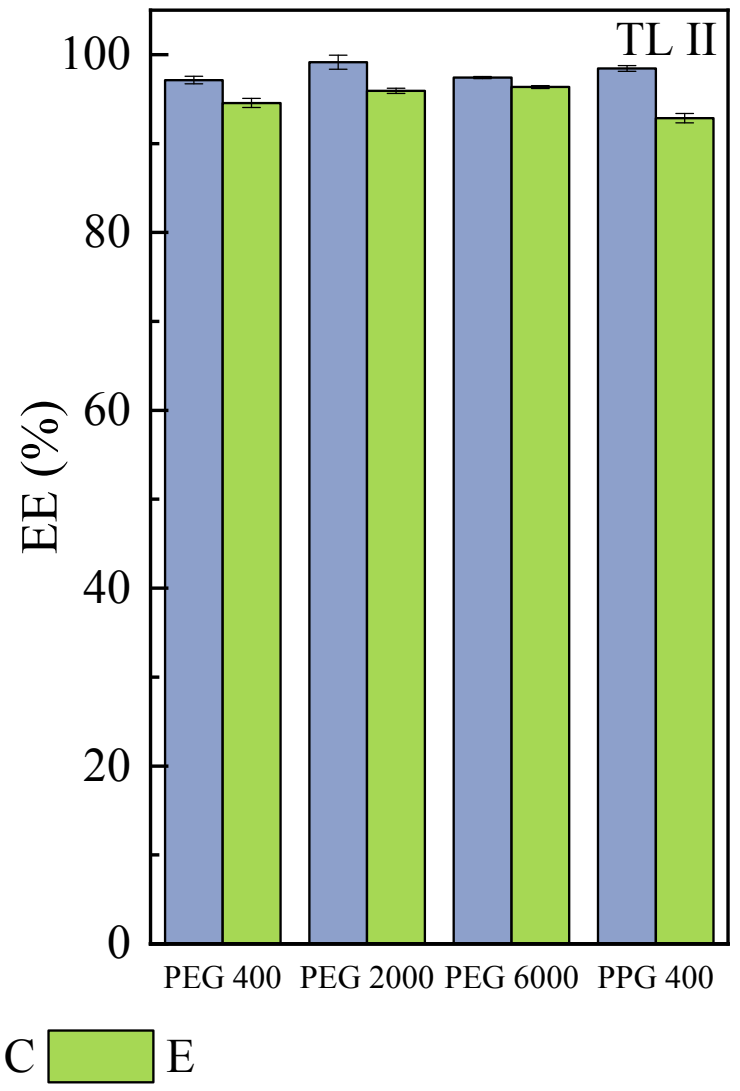

